\title{
Intergenic transcription causes repression by directing nucleosome assembly
}

\author{
Sarah J. Hainer, ${ }^{1}$ Justin A. Pruneski, ${ }^{1}$ Rachel D. Mitchell, Robin M. Monteverde, \\ and Joseph A. Martens ${ }^{2}$ \\ Department of Biological Sciences, University of Pittsburgh, Pittsburgh, Pennsylvania 15260, USA
}

\begin{abstract}
Transcription of non-protein-coding DNA (ncDNA) and its noncoding RNA (ncRNA) products are beginning to emerge as key regulators of gene expression. We previously identified a regulatory system in Saccharomyces cerevisiae whereby transcription of intergenic ncDNA (SRG1) represses transcription of an adjacent proteincoding gene (SER3) through transcription interference. We now provide evidence that $S R G 1$ transcription causes repression of $S E R 3$ by directing a high level of nucleosomes over $S R G 1$, which overlaps the SER3 promoter. Repression by $S R G 1$ transcription is dependent on the Spt6 and Spt16 transcription elongation factors. Significantly, spt6 and spt16 mutations reduce nucleosome levels over the SER3 promoter without reducing intergenic SRG1 transcription, strongly suggesting that nucleosome levels, not transcription levels, cause SER3 repression. Finally, we show that spt6 and spt16 mutations allow transcription factor access to the SER3 promoter. Our results raise the possibility that transcription of ncDNA may contribute to nucleosome positioning on a genome-wide scale where, in some cases, it negatively impacts protein-DNA interactions.
\end{abstract}

[Keywords: ncDNA; intergenic transcription; chromatin; repression]

Supplemental material is available for this article.

Received July 27, 2010; revised version accepted November 8, 2010.

Over the past decade, genome-wide expression studies in eukaryotes have revealed that transcription is not limited to protein-coding DNA, but rather occurs throughout entire genomes, often involving both DNA strands (Kapranov et al. 2007; Pheasant and Mattick 2007; Berretta and Morillon 2009; Jacquier 2009). Although the extent of transcription of non-protein-coding DNA (ncDNA) has been questioned recently (van Bakel et al. 2010), it is clear that eukaryotes produce many RNA molecules that do not encode proteins (noncoding RNAs [ncRNAs]) (Goodrich and Kugel 2009; Harrison et al. 2009; Mercer et al. 2009; Costa 2010). ncRNAs have diverse properties, ranging in size from short (microRNAs [miRNAs]) to long (long RNAs [lnRNAs]) and ranging in stability from stable to unstable. With the exception of several families of well-studied ncRNAs-including rRNAs, tRNAs, snRNAs, snoRNAs, and miRNAs - the biological functions of these ncRNAs are only beginning to be understood.

Although it is likely that some ncRNAs may represent transcriptional noise (Struhl 2007; Seila et al. 2009), it has become increasingly clear that transcription of noncoding regions of eukaryotic genomes plays important biological functions, primarily in regulating gene expression (Goodrich and Kugel 2009; Harrison et al. 2009; Mercer

\footnotetext{
${ }^{1}$ These authors contributed equally to this work.

${ }^{2}$ Corresponding author.

E-MAIL martens@pitt.edu; FAX (412) 624-4759.

Article published online ahead of print. Article and publication date are online at http://www.genesdev.org/cgi/doi/10.1101/gad.1975011.
}

et al. 2009). Examples of this include the Xist/Tsix RNAs involved in mammalian X inactivation (Lee 2009), the roX1 and roX2 RNAs involved in dosage compensation in Drosophila (Gelbart and Kuroda 2009), the human HOTAIR involved in the regulation of developmental genes (Rinn et al. 2007), the mouse Air and Kcnq1ot1 RNAs involved in establishing genomic imprinting (Royo and Cavaille 2008), and the mouse VL30 RNA and human PSF-binding ncRNAs that regulate cell proliferation and tumorigenesis (Li et al. 2009; Wang et al. 2009).

Significant advances have been made in understanding widely diverse mechanisms by which transcription of ncDNAs regulate gene expression. In some cases, it is the ncRNA product that regulates gene expression. ncRNAs have been shown to recruit complexes that modify chromatin, interact with activator and coactivator proteins and modulate their function, and interact with RNA polymerase II (Pol II) and other basal transcription factors to control their activity (Goodrich and Kugel 2009; Harrison et al. 2009; Mercer et al. 2009). Alternatively, the act of transcribing ncDNA has also been shown to both positively and negatively regulate gene expression. In most of these cases, a transcription interference mechanism has been proposed. Examples include mouse and human globin genes (Ashe et al. 1997; Gribnau et al. 2000); the Drosophila Hox genes (Schmitt et al. 2005; Mazo et al. 2007); and Saccharomyces cerevisiae SER3 (Martens et al. 2004), ADH1/ADH3 (Bird et al. 2006), IME4 (Hongay et al. 2006), and FLO11 (Bumgarner et al. 2009) genes. 
Although several mechanisms of transcription interference have been described, most involving RNA Pol II directly, experiments that distinguish between these mechanisms at specific genes have not been performed.

Interestingly, several studies in yeast have implied that transcription of ncDNA may contribute to gene regulation by altering chromatin structure. Transcription of a series of ncRNAs $5^{\prime}$ of the Schizosaccharomyces pombe $f b p 1^{+}$gene was found to facilitate an open chromatin conformation, allowing transcription factors access to the $f b p 1^{+}$promoter during glucose induction (Hirota et al. 2008). Antisense transcription has been shown to silence the expression of $\mathrm{PHO} 84$ by a mechanism that requires Hda1/2/3-dependent deacetylation of histones located at the PHO84 promoter (Camblong et al. 2007, 2009). Finally, two recent studies provide evidence that transcription of DNA antisense to the GAL10 gene alters post-translational modifications of histones that facilitate repression of the divergently transcribed GAL10 and GAL1 genes (Houseley et al. 2008; Pinskaya et al. 2009).

Previously, we showed that serine-dependent transcription of ncDNA (SRG1) in $S$. cerevisiae represses expression of the adjacent SER3 gene (Martens et al. 2004, 2005). In the presence of serine, transcription of SRG1 extends across the promoter of the adjacent SER3 gene, yielding two short transcripts that terminate 75 base pairs (bp) $5^{\prime}$ and $25 \mathrm{bp} \mathrm{3'}$ of the SER3 translational start (Thompson and Parker 2007), and a minor SRG1-SER3 readthrough transcript that extends to the end of SER3 (Martens et al. 2004; Thompson and Parker 2007). We provided evidence that it is the act of transcribing SRG1 across the SER3 promoter, rather than the SRG1 RNA products, that represses SER3 (Martens et al. 2004). In this study, we elucidate the mechanism whereby serine-dependent transcription of ncDNA (SRG1) in S. cerevisiae represses expression of the adjacent SER3 gene. We show that SER3 repression correlates with a broad region of strong micrococcal nuclease (MNase) protection spanning the entire $S R G 1$ transcription unit, suggesting that nucleosomes are loosely positioned across this region. Surprisingly, conditions that reduce $S R G 1$ transcription result in dramatically reduced MNase protection at the SER3 promoter, indicating a loss of nucleosome occupancy. By analyzing mutations in SPT6 and SPT16, two genes that encode subunits of the Spt6/Spn1(Iws1) and FACT elongation complexes, we provide evidence that it is the nucleosomes assembled at the SER3 promoter by intergenic SRG1 transcription, not RNA Pol II itself, that interfere with the binding of transcription factors to the SER3 promoter. Our data are consistent with a general model in which transcription of ncDNA can assemble nucleosomes that occlude DNA from binding by sequencespecific DNA-binding proteins.

\section{Results}

Evidence that nucleosomes occupy the SER3 promoter in repressing conditions

Previously, we showed that transcription of intergenic $S R G 1$ DNA is required for SER3 repression (Martens et al.
2004). Several pieces of data suggest that chromatin structure also plays an important role in SER3 repression. First, we identified histones and two activators of histone gene expression, Spt10 and Spt21 (Dollard et al. 1994; Hess et al. 2004; Eriksson et al. 2005), in a genetic screen for repressors of SER3 expression (J Pruneski, unpubl.). Second, DNA microarray experiments revealed that depletion of histone $\mathrm{H} 4$ resulted in strong SER3 derepression (Wyrick et al. 1999). Third, a mutation in SPT6, a gene that encodes a protein required to maintain proper chromatin structure over genes during transcription (Kaplan et al. 2003; Cheung et al. 2008), also results in SER3 derepression (Kaplan et al. 2003).

To investigate a possible role for chromatin structure in SER3 repression, we first determined the positions of nucleosomes across the SER3 locus in wild-type cells grown in SER3-repressing conditions (YPD) by a nucleosome scanning assay (Sekinger et al. 2005; Lee et al. 2007). Briefly, cells are treated with formaldehyde, spheroplasted, and then incubated with increasing amounts of MNase to digest nonnucleosomal DNA (see the Materials and Methods for details). As described previously (Brickner et al. 2007), we monitored MNase digestion of two sequences located in the GAL1-10 promoter-one within a well-positioned nucleosome (GAL1 NB), and one within an adjacent MNase-sensitive region (GAL1 NUB)-by quantitative PCR (qPCR) (Supplemental Fig. S1). DNA isolated from the MNase concentration where we observed significant protection of GAL1 NB relative to GAL1 NUB was then used to assess MNase protection across SRG1$S E R 3$. We performed qPCR with 38 unique primer pairs to amplify overlapping SRG1-SER3 sequences (Fig. 1A) from both MNase-digested and undigested DNA. MNase protection for each of these sequences was quantified as the ratio of template present in MNase-digested DNA over undigested DNA that was then normalized to the amount of MNase-protected GAL1 NB template. Using this method, we identified peaks of MNase protection, indicating the presence of a positioned nucleosome at the $3^{\prime}$ end of AIM9 (the gene adjacent to SRG1) and two at the $5^{\prime}$ end of the SER3 ORF (Fig. 1B). We also found a 200-bp MNasesensitive region (from -750 to -550 with respect to the SER3 ATG) corresponding to the SRG1 promoter, indicating a nucleosome-depleted region that is a hallmark of many yeast promoters (Yuan et al. 2005; Albert et al. 2007; Lee et al. 2007). In addition, we identified a broad region of MNase protection that begins at the SRG1 transcription start site $(-475)$ and extends across the SER3 promoter to the SER3 translational start site, a region that defines the SRG1 transcription unit. This pattern of strong MNase protection implies the presence of nucleosomes that are positioned randomly across the SRG1 transcription unit. Therefore, the SER3 promoter lacks the typical nucleosome-depleted region (Yuan et al. 2005; Albert et al. 2007; Lee et al. 2007). These results are consistent with our previously reported indirect-labeling experiments (Martens and Winston 2002) and with genome-wide nucleosome positioning experiments (Lee et al. 2007).

To determine if SRG1 transcription affects the chromatin structure at $S E R 3$, we repeated the nucleosome 
A

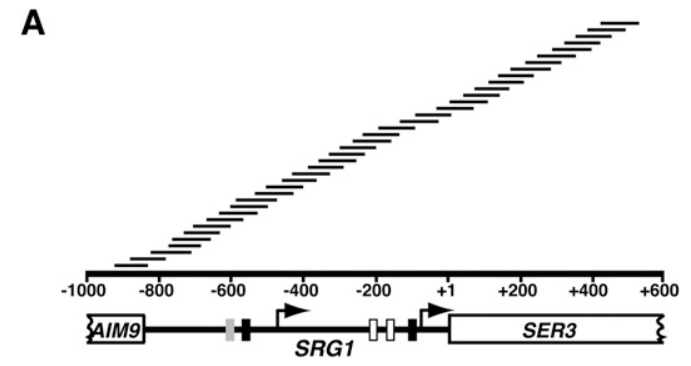

B
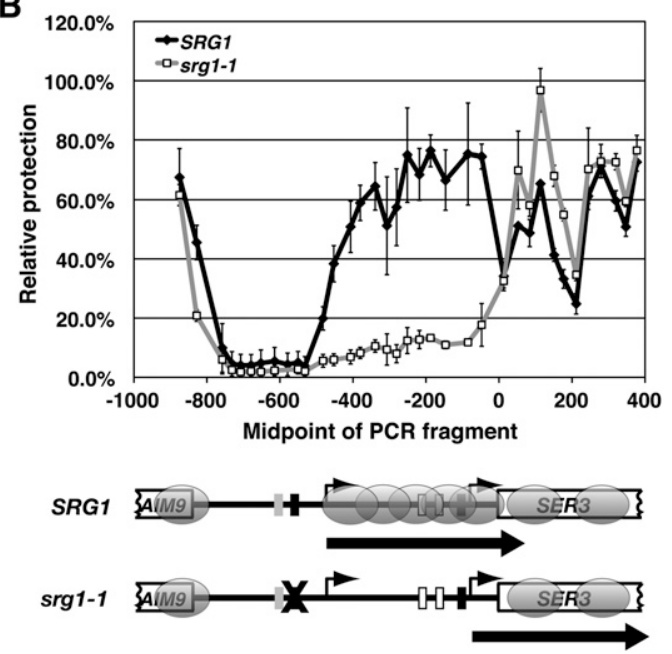

Figure 1. Nucleosome positions and relative occupancy at $S E R 3$ in the presence and absence of SRG1 transcription. (A) Schematic of SER3 locus, including the 3' $161 \mathrm{bp}$ of AIM9 $\left(-1000\right.$ to -839 relative to SER3 ATG) and the $5^{\prime} 600 \mathrm{bp}$ of the SER3 ORF. The arrows at -475 and -75 indicate the transcription start sites of SRG1 and SER3, respectively. Blocks of intergenic sequence identity between $S$. cerevisiae and four related yeast strains are marked, including the SRG1 and SER3 TATAs (black boxes), sequences required for SER3 activation (white boxes), and a Cha4-binding site (gray box). The scale represents the distance from the SER3 translation start $(+1)$. The tiled black bars above the scale indicate the DNA fragments amplified by qPCR to quantify nucleosome position and relative occupancy (see Supplemental Table S2 for details). (B) Nucleosome scanning assay was performed on wild-type (FY4, FY2097, and FY1350) and srg1-1 (YJ582, FY2250, and YJ585) cells that were grown in YPD medium (SER3 repressed) at $30^{\circ} \mathrm{C}$. Using qPCR, the relative MNase protection of each SER3 template was calculated as a ratio to the control GAL1 NB template found within a well-positioned nucleosome in the GAL1-10 promoter (see Supplemental Fig. S1). Each point on the graph shows the mean \pm SEM from three independent experiments that are plotted at the midpoint of each PCR product. Results for amplicons SER3-5 to SER3-41 are shown. Below the graph, a diagram of the SER3 locus indicates the positions of nucleosomes (gray ovals) extrapolated from the MNase protection data. The block arrows indicate the transcription activity of SRG1 and SER3, respectively. srg1-1 strains have a mutated TATA sequence (marked by an $\mathrm{X}$ ) that inhibits $S R G 1$ transcription, causing SER3 derepression.

scanning assay using $\operatorname{srg} 1-1$ strains, which carry a mutation of the SRG1 TATA sequence. This mutation severely reduces $S R G 1$ transcription, resulting in strong derepres- sion of SER3 (Martens et al. 2004). In the srg1-1 cells, MNase protection was reduced specifically over the SRG1 transcription unit as compared with wild-type cells, indicating a dramatic loss of nucleosome occupancy (Fig. 1B). Our results reveal a positive correlation between SRG1 transcription and nucleosome occupancy across $S R G 1$, an unexpected finding given the negative correlation between transcription and nucleosome occupancy generally observed for protein-coding genes (Lee et al. 2004; Schwabish and Struhl 2004).

\section{Serine-dependent transcription of SRG1 intergenic DNA controls nucleosome occupancy of the SER3 promoter}

We showed previously that SER3 expression is tightly controlled by the serine-dependent regulation of SRG1 transcription (Martens et al. 2005). Therefore, we also measured MNase accessibility at SER3 in wild-type strains that were grown in synthetic complete (SC) + serine (SRG1 induced; SER3 repressed) and then shifted to SC - serine (SRG1 repressed, SER3 induced) for 25 min. Since the extent of the MNase digestion of the GAL1 NB region was identical in these different growth conditions (Supplemental Fig. S2), we again normalized all SER3 data to this region. As expected for cells grown in serine-rich media, the relative MNase protection across SRG1-SER3 is nearly identical to that observed for cells grown in YPD (cf. wild-type strains in Figs. 1B, 2A). When cells were shifted to media lacking serine, we measured a significant decrease in MNase protection over the SRG1 transcribed region. However, rather than extending across the entire $S R G 1$ transcription unit, as was observed for $\operatorname{srg} 1-1$, the reduced MNase protection was restricted to a 200-bp region that included sequences that had been determined previously to be required for SER3 activation (Martens et al. 2004). An MNase-protected region of $\sim 350 \mathrm{bp}$, consistent with two closely associated nucleosomes or possibly one nucleosome that adopts multiple positions, remains near the $5^{\prime}$ end of SRG1. This MNase-protected region begins at a more 5' position, including the SRG1 transcription start site and possibly the SRG1 TATA, as compared with the beginning of the broad peak of MNase protection that was measured for cells grown in serine-rich media. Thus, in contrast to the complete loss of nucleosomes across SRG1 that occurs in the $\operatorname{srg} 1-1$ strains, serine starvation depletes nucleosomes specifically over sequences required for SER3 activation. Therefore, in response to serine starvation, the SER3 promoter adopts the typical promoter architecture, with +1 and -1 nucleosomes flanking a nucleosome-depleted UAS (Albert et al. 2007; Lee et al. 2007).

To determine if the loss of nucleosome occupancy at the SER3 promoter is caused by a loss of SRG1 transcription and is not simply an effect of the resulting increase in SER3 transcription, we repeated the nucleosome scanning assay using strains that contain a mutation in the SER3 TATA sequence (ser3-100). Although the ser3-100 mutation strongly inhibits SER3 activation when cells 
A
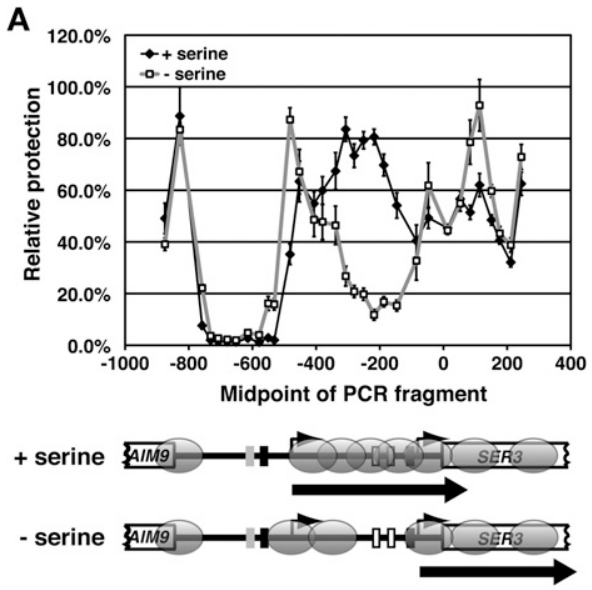

B

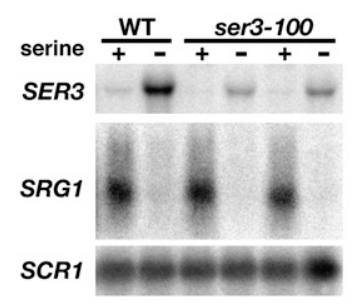

C

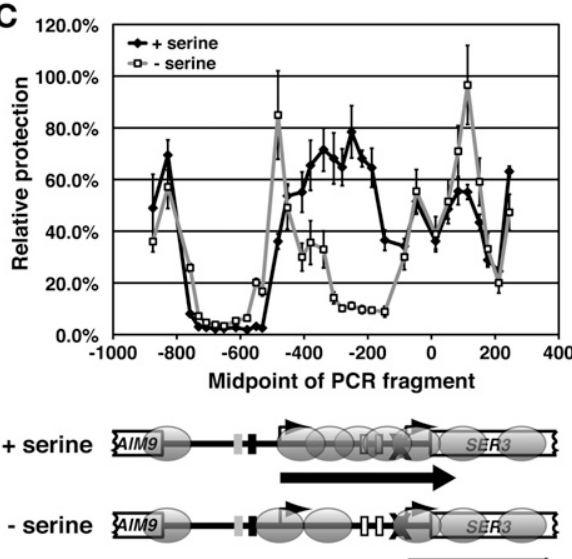

Figure 2. Effect of serine on nucleosome positions and relative occupancy at SER3. (A) Nucleosome scanning assay was performed on wild-type cells (FY2097 and FY4) that were grown at $30^{\circ} \mathrm{C}$ in SC + serine media (+ serine) and then shifted to SC serine media (- serine) for $25 \mathrm{~min}$ as described in Figure 1. Each point on the graph shows the mean relative MNase protection \pm SEM from four independent experiments (two for each strain) plotted at the midpoint of each PCR product. Results for amplicons SER3-7 to SER3-41 are shown. (B) Northern analysis of SER3 and SRG1 was performed on a wild-type (FY2097) and two ser3-100 strains (YJ275 and FY2099) that have a mutated SER3 TATA. Cells were grown at $30^{\circ} \mathrm{C}$ in $\mathrm{SC}+$ serine media (+ serine) and then shifted to SC - serine media (- serine) for 25 min. SCR1 serves as a loading control. (C) Nucleosome scanning assay was performed on ser3-100 strains (YJ275 and FY2099) as described in $A$.

are shifted from serine-rich to serine starvation media (10-fold decrease in SER3 mRNA levels) (Fig. 2B), the changes in MNase protection between these growth conditions were identical to those observed for a wild type (Fig. 2, cf. A and C). Therefore, reduced nucleosome occupancy over the $S E R 3$ promoter is not a consequence of increased SER3 expression.

\section{FACT and Spt6/Spn1(Iws1) are required to repress SER3}

Our results thus far are consistent with two possible mechanisms for transcription interference at SER3. In the first possibility, similar to the conventional transcription interference mechanism (Greger et al. 2000), RNA Pol II elongating across SRG1 competes with transcription factors for binding to the SER3 promoter. In the second possibility, the nucleosomes maintained over the SER3 promoter by SRG1 transcription compete with transcription factor access to the SER3 promoter. If the latter possibility is true, we reasoned that disrupting nucleosome reassembly during transcription might cause SER3 derepression. Several studies have implicated the essential, highly conserved FACT and Spt6/Spn1(Iws1) transcription elongation complexes in transcription-dependent chromatin reassembly (Belotserkovskaya et al. 2003; Kaplan et al. 2003; Mason and Struhl 2003; Cheung et al. 2008; Jamai et al. 2009). Northern analyses were performed on several temperature-sensitive mutants of the Spt6/Spn1(Iws1) and FACT complexes that were grown in YPD at permissive $\left(30^{\circ} \mathrm{C}\right)$ and nonpermissive $\left(37^{\circ} \mathrm{C}\right)$ temperatures. Large increases in SER3 mRNA levels were detected in multiple spt6 and spn1(iws1) mutants at both $30^{\circ} \mathrm{C}$ and $37^{\circ} \mathrm{C}$ (Fig. 3A). While increases were more modest and variable in the FACT mutants (spt16, pob3, and $n h p 6$ ), we did find that, in at least one mutant, spt16197, a significant increase in SER3 mRNA levels occurred at $30^{\circ} \mathrm{C}$ (Fig. 3B). Importantly, SRG1 RNA levels were not significantly reduced in most of the mutant strains as compared with a wild type at $30^{\circ} \mathrm{C}$.

We also performed chromatin immunoprecipitation (ChIP) experiments to measure RNA Pol II occupancy across the SRG1/SER3 locus in a wild-type strain and two of these mutants (spt6-1004 and spt16-197) that express either untagged Rpb1 (control) or a myc-tagged version of Rpb1 (Rpb1-13myc). The spt6-1004 and spt16-197 mutants have both been well characterized and share similar phenotypes characteristic of transcription defects, including sensitivity to the nucleotide analog 6-azauracil, suppression of Ty insertions, and cryptic intragenic transcription (Kaplan et al. 2003; Mason and Struhl 2003). Consistent with our Northern data, RNA Pol II strongly associates with the $S R G 1$ transcription unit (Fig. 3C) to similar levels in wild-type, spt6-1004, and spt16197 cells. Taken together, these results show that SER3 repression is strongly dependent on both Spt6/Spn1(Iws1) and FACT. When these factors are mutated, SER3 is derepressed without affecting RNA Pol II levels at $S R G 1$. This result argues against a model in which it is the level of active transcription that confers transcription interference.

Beyond the primary sites of $S R G 1$ transcription termination, we found a twofold increase in RNA Pol II 


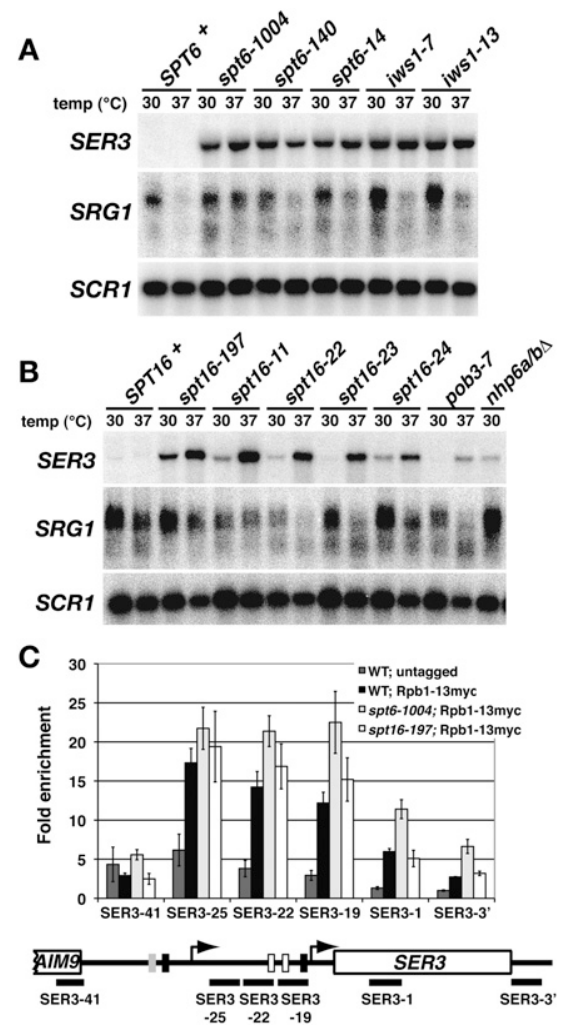

Figure 3. Repression of $S E R 3$ is dependent on Spt6/Spn1(Iws1) and the FACT complex. (A) Northern analysis of SER3, SRG1, and SCR1 (loading control) was performed on wild-type (FY4), spt6-1004 (FY2425), spt6-140 (FY111), spt6-14 (FY1221), iws1-7 (GHY1199), and iws1-13 (GHY1200) strains. Cells were grown in YPD at $30^{\circ} \mathrm{C}$ to mid-log and then shifted for $60 \mathrm{~min}$ to $37^{\circ} \mathrm{C}$. (B) Northern analysis of SER3, SRG1, and SCR1 (loading control) was performed on wild-type (FY4), spt16-197 (FY346), spt16-11 (TF8030-1), spt16-22 (YJ832), spt16-23 (YJ833), spt16-24 (TF7783-24), pob3-7 (TF8031-1), and nhp6as::URA3 nhp6bs:: URA3 (FY1411) strains that were grown in YPD. (C) ChIP analysis was performed on chromatin isolated from wild-type (YJ877, YJ878, YJ879, and YJ884), spt6-1004 (YJ886, YJ887, YJ888, and YJ892), and spt16-197 (YJ841, YJ842, and YJ843) strains expressing Rpb1-C13myc and untagged control strains (FY4, FY5, and YJ586). Rpb1-C13myc was immunoprecipitated with $\alpha$-myc A14 antibody from chromatin prepared from cells that were grown in YPD at $30^{\circ} \mathrm{C}$. The amount of immunoprecipitated DNA was determined by qPCR as a percentage of the input material and is expressed as the fold enrichment over a control region of chromosome $V$ that lacks ORFs (Supplemental Table S2, No ORF). Each bar represents the mean \pm SEM from at least three independent experiments. Below the graph is a schematic of SER3 with black bars corresponding to the regions amplified by qPCR (see Supplemental Table S2 for details).

occupancy in the spt6-1004 cells as compared with wildtype cells, which is consistent with our Northern data (Fig. 3C). However, we did not detect an increase in RNA Pol II in the spt16-197 cells. Although surprising given the increase in SER3 mRNA levels in this mutant, this result may be reconciled if we consider that SRG1 transcription does not always terminate properly, result- ing in the production of a minor readthrough that extends to the end of SER3 (Martens et al. 2004; Thompson and Parker 2007). Importantly, we found that the level of SRG1-SER3 readthrough product is reduced in both spt61004 and spt16-197 mutants (S Hainer, unpubl.), which is likely due to increased initiation at the SER3 promoter. Therefore, increased RNA Pol II occupancy in these mutant strains that would better reflect the observed increases in SER3 transcription are likely masked by the RNA Pol II that occupies $S E R 3$ as a result of the synthesis of an SRG1-SER3 readthrough product.

\section{Nucleosome occupancy of the SER3 promoter is reduced in spt6-1004 and spt16-197 mutants at the permissive temperature}

To test whether the level of nucleosomes over SRG1 affects SER3 repression, we next performed nucleosome scanning assays to compare MNase accessibility across SRG1 in wild-type, spt6-1004, and spt16-197 cells that were grown in $\mathrm{YPD}$ at $30^{\circ} \mathrm{C}$. We again normalized MNase protection of each SRG1-SER3 region to the GAL1 NB region, as the MNase accessibility of the GAL1 control regions was indistinguishable between these strains (Supplemental Fig. S3A). Compared with wild-type cells, we measured a significant reduction of MNase protection specifically across the SRG1 transcribed unit in spt6-1004 cells (fourfold decrease) and to a slightly lesser extent in spt16-197 cells (threefold decrease) (Fig. 4A), indicating nucleosome depletion across SRG1. These results are strikingly similar to the nucleosome scanning results we obtained for the srg1-1 mutant (Fig. 1B). However, while SRG1 transcription was greatly reduced in $\operatorname{srg} 1-1$ strains, it remained at wild-type levels in the spt6-1004 and spt16-197 mutants.

To complement our MNase experiments, we performed histone H3 ChIP assays in these same strains grown under the same conditions (Fig. 4B). In wild-type cells, we detected significant histone $\mathrm{H} 3$ occupancy over the SER3 promoter as compared with the SRG1 promoter, which is consistent with nucleosomes occupying the SER3 promoter. Moreover, at least for spt6-1004 cells, there is a twofold to threefold decrease in histone $\mathrm{H} 3$ occupancy specifically over the SER3 promoter that parallels the increase in MNase sensitivity over this region. Curiously, we did not observe a similar decrease in histone H3 occupancy over the SER3 promoter in spt16-197 cells. Since the loss of MNase protection is less pronounced in the spt16-197 mutants as compared with the spt6-1004 mutants, it is possible that histone $\mathrm{H} 3$ $\mathrm{ChIP}$ is not sensitive enough to detect a change in histone occupancy between wild-type and spt16-197 strains. Alternatively, nucleosomes may only partially reassemble in the spt16-197 mutant in a manner that makes them more accessible to MNase without altering histone $\mathrm{H} 3$ occupancy. Based on previous studies (Belotserkovskaya et al. 2003; Xin et al. 2009), an intriguing possibility is that reassembly of the $\mathrm{H} 2 \mathrm{~A} / \mathrm{H} 2 \mathrm{~B}$ dimers at the SER3 promoter may be specifically reduced by the spt16-197 mutation. Taken together, these data support a model 
A

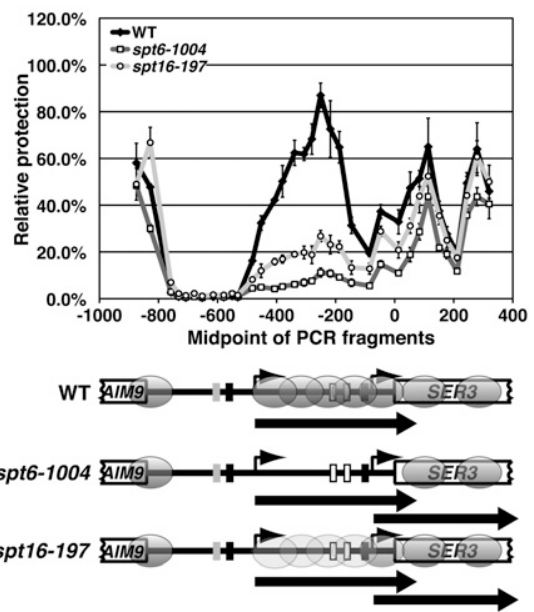

B

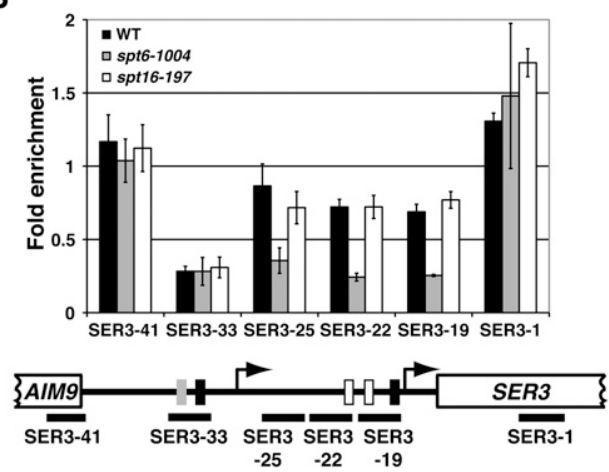

Figure 4. Nucleosome positions and relative occupancy at SER3 in spt6-1004 and spt16-197 mutants. (A) Nucleosome scanning assay was performed on wild-type (FY2134, YJ864, and YJ847), spt6-1004 (FY2180, YJ855, YJ862), and spt16-197 (FY346, YJ859, and YJ916) strains that were grown in YPD at $30^{\circ} \mathrm{C}$ as described in Figure 1. The light-gray ovals over the SRG1 transcription unit in the spt16-197 strain reflect that this region is slightly more protected from MNase digestion as compared with the spt6-1004 strain. (B) Histone H3 ChIP was performed on chromatin isolated from wild-type (FY4, FY5, and YJ586), spt6-1004 (YJ886, YJ887, and YJ888), and spt16-197 (YJ844, YJ845, and YJ846) cells that were grown in YPD. The amount of immunoprecipitated DNA was determined by qPCR as a percentage of the input material and is expressed as the fold enrichment over GAL1 NB (see Supplemental Fig. S1). Each bar represents the mean \pm SEM of at least three independent experiments. Below the graph is a schematic of SER3 with black bars corresponding to the regions amplified by qPCR (see Supplemental Table S2 for details).

whereby FACT and Spt6/Spn1(Iws1) are required for SRG1 transcription-dependent assembly of nucleosomes that repress SER3.

spt6-1004 and spt16-197 mutants are defective for transcription interference at SER3

To test whether SRG1 transcription-dependent nucleosomes interfere with transcription factor binding to the SER3 promoter, we performed ChIP experiments in spt61004 and spt16-197 mutants. Because sequence-specific activators of SER3 remain unknown, we first used a previously described ser3::GAL7UAS allele in which the putative $S E R 3$ UAS is replaced with two binding sites for the Gal4 transcription activator (Martens et al. 2004). We then measured Gal4 occupancy by ChIP in wild-type, srg1-1, spt6-1004, and spt16-197 strains that all contain the ser3::GAL7UAS allele and were grown in YPgal (Fig. 5A). Consistent with our previous data (Martens et al. 2004), Gal4 occupancy at the SER3 promoter increases eightfold in the $\operatorname{srg1-1}$ control strain where $S R G 1$ is no longer transcribed and the SER3 promoter is depleted of

\section{A}
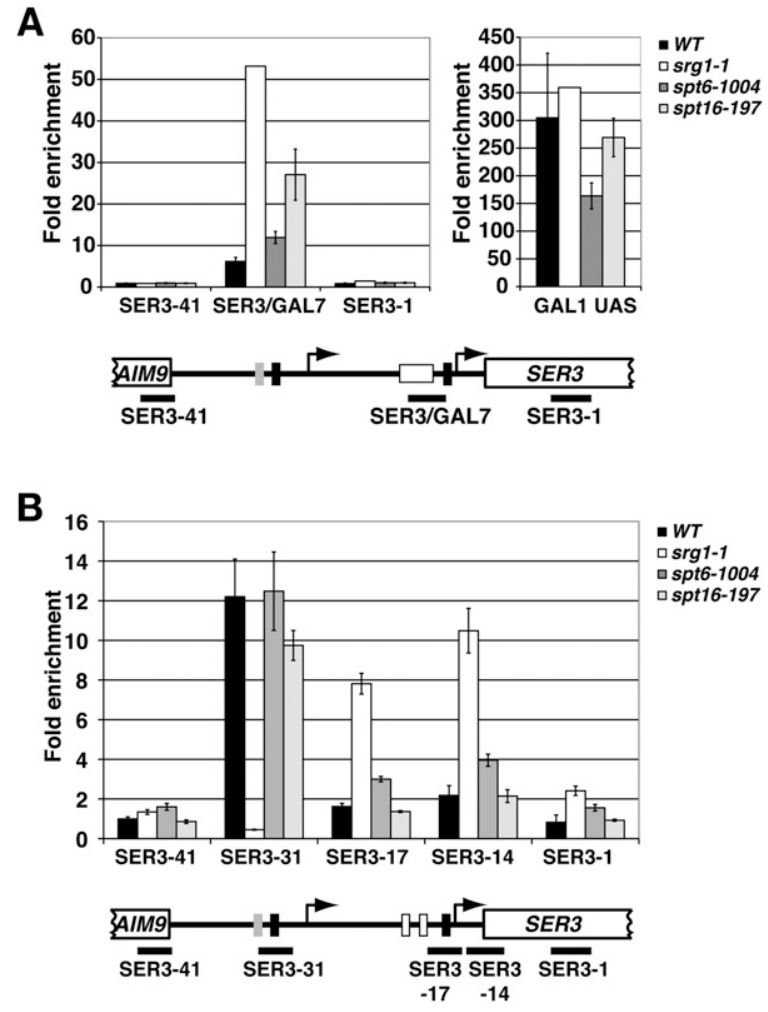

Figure 5. spt6-1004 and spt16-197 mutants are defective for transcription interference at SER3. (A) Gal4 ChIP was performed on wild-type (YJ871, YJ872, and YJ873), spt6-1004 (YJ875, YJ876, and YJ850), spt16-197 (YJ867, YJ868, and YJ869), and positive control srg1-1 (FY2260) cells that all contain the ser3::GA$L 7 U A S$ allele. Chromatin was prepared from cells grown at $30^{\circ} \mathrm{C}$ in YPraf to $0.8 \times 10^{7}$ cells per milliliter, and then for an additional $4 \mathrm{~h}$ at $30^{\circ} \mathrm{C}$ after the addition of $2 \%$ galactose. Gal4 ChIP signals were determined by qPCR at the three SER3 locations (left histogram), and at GAL1 as a positive control (right histogram). All values were normalized to a control region located near the telomere of chromosome VI (TELVI) (Supplemental Table S2) and represent the mean \pm SEM. Below the graph is a diagram of the ser $3:: G A L 7 U A S$ allele in which the putative SER3 UAS region was replaced with the GAL7 UAS region containing two Gal4-binding sites (white box). The black bars indicate the regions of SER3 amplified by qPCR. (B) TBP ChIP was performed on chromatin isolated from wild-type (FY4, FY5, YJ586, and KY719), spt6-1004 (YJ886, YJ887, YJ888, and YJ892), spt16-197 (YJ841, YJ842, YJ843, and YJ844), and positive control srg1-1 (FY2471, YJ582, YJ583, and YJ585) strains that were grown in YPD at $30^{\circ} \mathrm{C}$ as described in Figure 3C. 
nucleosomes. In the spt6-1004 and spt16-197 strains where $S R G 1$ is transcribed at wild-type levels but nucleosome occupancy at the SER3 promoter is reduced, Gal4 occupancy at the SER3 promoter was also increased twofold and fourfold, respectively (Fig. 5A, left panel). Based on our SER3 expression and nucleosome occupancy data (Figs. 3A, 4A), the twofold increase in Gal4 occupancy at the SER3 promoter in the spt6-1004 strains was lower than expected. However, this result is likely related to the fact that we also found reduced Gal4 occupancy at the control GAL1 promoter in spt6-1004 cells as compared with wildtype, srg1-1, and spt16-197 cells (Fig. 5A, right panel).

We also compared TBP occupancy by ChIP at the SRG1 and SER3 TATA sequences in wild-type, srg1-1, spt61004, and spt16-197 strains that contain the endogenous SRG1-SER3 locus (Fig. 5B). The SRG1 and SER3 TATA sequences are both conserved among related yeast strains, bind TBP, and are required for SRG1 and SER3 transcription, respectively (Martens and Winston 2002; Martens et al. 2004). At the SRG1 TATA, there is little difference in TBP occupancy in the spt6-1004 and spt16-197 mutants as compared with the wild-type strains, which agrees with our Northern and RNA Pol II ChIP data (see Fig. 3). At the SER3 TATA, TBP occupancy increased twofold in spt61004 cells as compared with a fourfold increase in srg1-1 control cells, suggesting that the loss of nucleosomes over the SER3 promoter in the spt6-1004 mutants either increases TBP binding directly or possibly indirectly by allowing an unknown SER3 activator protein better access to the SER3 promoter. Interestingly, we did not observe a significant difference in TBP occupancy in the spt16-197 mutant. This result may not be surprising, considering the increase in SER3 expression is significantly lower in this mutant as compared with the spt6-1004 mutant (Fig. 3; see Supplemental Fig. S4B for a direct comparison). Therefore, this assay may lack the sensitivity to detect a significant difference in TBP occupancy between wild-type and spt16197 cells.

From these data, we conclude that transcription interference at SER3 is dependent, at least in part, on Spt6 and Spt16. Taken together with results described earlier, our findings suggest that transcription interference of SER3 is partially mediated by nucleosomes that occupy the $S E R 3$ promoter as a consequence of $S R G 1$ transcription from intergenic DNA.

\section{Histone modifications that suppress cryptic intragenic transcription are not required for SER3 repression}

Spt6 and Spt16 have been shown previously to suppress transcription initiation from cryptic promoters that are located within protein-coding regions (Mason and Struhl 2003; Kaplan et al. 2009). Cryptic intragenic transcription is also suppressed by a cascade of transcriptiondependent post-translational histone modifications (Lee and Shilatifard 2007; Li et al. 2007a). During transcription, Set2 methylates Lys 36 of histone H3, thereby marking nucleosomes associated with recently transcribed DNA (Pokholok et al. 2005; Rao et al. 2005). Dimethylated H3K36 acts as a binding site for the Rpd3S histone deacetylase complex (Youdell et al. 2008). Upon recruitment, Rpd3S deacetylates the reassembled nucleosomes on the N-terminal tails of histones $\mathrm{H} 3$ and $\mathrm{H} 4$, which suppresses cryptic intragenic transcription, presumably by occluding transcription factor access (Carrozza et al. 2005; Joshi and Struhl 2005; Keogh et al. 2005). Recently, Set1-dependent methylation of H3K4 has also been implicated as a signal for transcription-dependent histone deacetylation by Rpd3S (Pinskaya et al. 2009) and the Set3 complex (Kim and Buratowski 2009). Because of these observations, a likely hypothesis is that Set1 and Set2 may contribute to $S E R 3$ repression by regulating similar histone modifications over the SER3 promoter in response to SRG1 transcription. To test this possibility, we performed a Northern analysis to measure the effect of deleting the genes encoding the Set1, Set2, and Dot1 histone methyltransferases; the Rco1 subunit of Rpd3S; and the Set3 subunit of the Set3 complex on SER3 and $S R G 1$ expression. Deletions of any one of these genes or a set $1 \Delta$ set2 $2 \Delta$ double deletion has no effect on SER3 or SRG1 mRNA levels (Fig. 6). Moreover, mutations of histone H3 Lys 4 (methylated by Set1), Lys 36 (methylated by Set2), or Lys 79 (methylated by Dot1) also has little to no effect on SER3 repression (S Hainer, unpubl.). Therefore, our results suggest that the relative contribution of these histone reassembly mechanisms may vary at different loci throughout the genome.

\section{Discussion}

In this study, we provide evidence that intergenic transcription represses adjacent gene transcription by assembling a repressive chromatin structure, rather than by the act of transcription. First, we showed that SRG1 intergenic transcription is required not only for repression of the adjacent SER3 gene, but also to maintain MNase protection of the SER3 promoter. Second, we determined that changes in the MNase protection of the SER3 promoter are caused by changes in SRG1 transcription and are not an effect of the changes to SER3 transcription. Third, we found that cells expressing mutant versions of the Spt6 and Spt16 elongation factors derepress SER3 and reduce MNase protection across the SER3 promoter

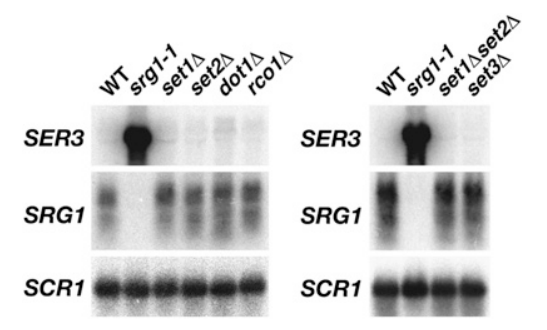

Figure 6. Repression of $S E R 3$ does not require histone methyltransferases or the Rpd3S and Set3C histone deacetylase complexes. Northern analysis of SER3, SRG1, and SCR1 (loading control) was performed on wild-type (YJ586), srg1-1

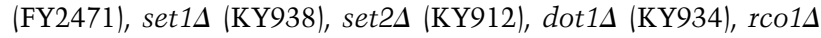

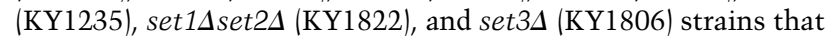
were grown in $\mathrm{YPD}$ at $30^{\circ} \mathrm{C}$. 
without altering SRG1 RNA levels or RNA Pol II occupancy across $S R G 1$. These results clearly implicate the nucleosomes assembled on the $S E R 3$ promoter as the key factor in SER3 repression. Finally, we found that Spt6 and Spt16 are required to inhibit transcription factor binding to the SER3 promoter, which suggests that the nucleosomes assembled at the SER3 promoter by these factors interfere with the binding of transcription factors to their sites on DNA.

Taken together with our previous studies (Martens and Winston 2002; Martens et al. 2004, 2005), we propose the following model for SER3 regulation (Fig. 7). When cells are grown in serine-rich medium, the Cha4 DNA-binding protein recruits the Swi/Snf and SAGA complexes, resulting in the induction of $S R G 1$ transcription. RNA Pol II transcribes SRG1 across the SER3 promoter, disassembling nucleosomes in its path and then reassembling them in its wake by a mechanism that involves both Spt6 and Spt16. SRG1 transcription is thus required to maintain nucleosomes across the SER3 promoter, interfering with transcription factor binding. When cells are then transferred to serine starvation conditions, Cha4 no longer recruits Swi/Snf and SAGA, resulting in decreased $S R G 1$ transcription. Without intergenic transcription to maintain them, nucleosomes are depleted over the SER3 UAS, allowing transcription factors-either an as yet unknown site-specific DNA-binding activator or possibly TBP and RNA Pol II-to bind and activate SER3. Two positioned nucleosomes remain at the $5^{\prime}$ end of SRG1, where they are likely to inhibit $S R G 1$ transcription.

In addition to its role in nucleosome assembly during transcription, Spt6 has also been reported to reassemble

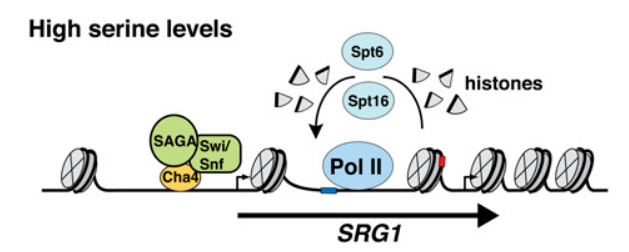

Serine starvation

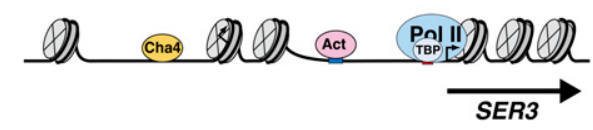

Figure 7. A model for SER3 regulation by SRG1 intergenic transcription. When serine is available to the cells, DNA-bound Cha4 recruits SAGA and Swi/Snf to initiate SRG1 transcription, possibly by remodeling the two nucleosomes located at the 5 ' end of $S R G 1$ to expose the SRG1 transcription start site. RNA Pol II transcribes SRG1 and, through Spt6 and Spt16, disassembles nucleosomes in its path and then reassembles them in its wake. As a result, nucleosomes continuously occupy the SER3 UAS where they repress $S E R 3$ by occluding the $S E R 3$ promoter from transcription factor binding. In the absence of serine, SRG1 transcription is repressed, possibly due to the presence of two nucleosomes at its $5^{\prime}$ end that encompass its transcription start site. In the absence of SRG1 transcription, the SER3 UAS is depleted of nucleosomes, allowing an as yet unknown activator (Act) and/or TBP and RNA Pol II to bind and activate SER3 transcription. nucleosomes at the promoters of $\mathrm{PHO} 5$ and several other yeast genes during repression (Adkins and Tyler 2006). Therefore, an alternative model for SER3 repression is that Spt6 and, possibly, Spt16 reassemble nucleosomes over the SER3 promoter independently of SRG1 transcription. Thus, mutations in these factors may bypass the normal role for $S R G 1$ transcription, which is to interfere with the recruitment of chromatin remodeling factors needed to displace the repressive nucleosomes at the SER3 promoter. A prediction of this model is that the increased levels of SER3 expression observed in the spt61004 and spt16-197 mutants would no longer be dependent on sequence-specific activators to recruit chromatin remodeling factors, analogous to what has been observed for PHO5 (Adkins and Tyler 2006). To test this alternative model, we first identified a $37-$ bp sequence within the SER3 promoter (from -192 to -228 ; SER3 ATG $=+1$ ) that is required for $S E R 3$ activation in response to serine starvation (Supplemental Fig. S4A). When this sequence was deleted in the spt6-1004 and spt16-197 strains, SER3 mRNA levels were reduced as compared with similar strains expressing wild-type SER3 (Supplemental Fig. S4B). Therefore, spt6-1004 and spt16-197 mutations do not bypass the requirement of the SER3 UAS for SER3 activation, which argues against this alternative model.

Although MNase accessibility has been used extensively to predict nucleosome occupancy in eukaryotic organisms (for examples, see Yuan et al. 2005; Lee et al. 2007), we cannot rule out the possibility that DNA-binding proteins may contribute to the protection of the SER3 promoter from MNase digestion in serine-rich conditions. However, our observation that MNase protection over the SER3 promoter was reduced in spt6-1004 and spt16-197 mutants without affecting RNA Pol II occupancy suggests that at least RNA Pol II and its associated factors do not affect MNase digestion.

If $S R G 1$ transcription from intergenic DNA is required to maintain nucleosomes over the SER3 UAS, then from where might these nucleosomes originate? An intriguing source of these nucleosomes would be those positioned over the SRG1 transcription start site and TATA (Fig. 7) that likely inhibit SRG1 transcription in the absence of serine. Based on this study and our previous work (Martens and Winston 2002; Martens et al. 2004, 2005), Swi/Snf, when recruited to the SRG1 promoter in response to serine, may slide these nucleosomes toward SER3 to facilitate preinitiation complex assembly and $S R G 1$ transcription. Once RNA Pol II begins to transcribe $S R G 1$, the nucleosomes originally moved by Swi/Snf are disassembled to allow passage of RNA Pol II, and then are reassembled behind RNA Pol II by Spt6 and Spt16. Therefore, the activities of Swi/Snf, Spt6/Spn1, and FACT may combine to establish and maintain nucleosomes over the SER3 promoter that interfere with transcription factor binding to this region. This scenario would also explain the difference in nucleosome occupancy at the $5^{\prime}$ end of SRG1 observed for wild-type cells grown in the serine starvation media as compared with $\operatorname{srg} 1-1$ cells grown in serine-rich media, two conditions in which 
SER3 is strongly derepressed (Figs. 1, 2A). In contrast to wild-type cells grown in serine starvation medium, where it is no longer recruited, Swi/Snf is presumably still recruited by Cha4 in the srg1-1 (SRG1 TATA mutant) cells that are grown in serine-rich media. Thus, Swi/Snf can remodel the nucleosomes at the $5^{\prime}$ end of SRG1; however, these nucleosomes cannot be maintained in the absence of SRG1 transcription.

In addition to the nucleosome reassembly activity of Spt6/Spn1 and FACT, it has been well documented that a cascade of transcription-dependent post-translational modifications of histones found within nucleosomes over protein-coding genes contributes to the repression of intragenic transcription initiation (Lee and Shilatifard 2007; Li et al. 2007a). However, our studies show that SER3 repression appears to be independent of at least some of these marks, including Set1-mediated methylation of histone H3 K4, Set2-mediated methylation of K36, and the removal of histone $\mathrm{H} 3$ and $\mathrm{H} 4$ acetylation by the Rpd3S and Set3C histone deacetylase complexes. Although we cannot rule out the possibility that other post-translational histone modifications may be involved, our results indicate a difference in the requirement of transcription-dependent post-translational histone modifications between $S E R 3$ repression by $S R G 1$ transcription and repression of cryptic intragenic transcription. This difference may be related to the fact that $S R G 1$ is a relatively short transcription unit $(\sim 400 \mathrm{bp})$ that is highly transcribed. It has been reported recently that cryptic intragenic transcription preferentially occurs at lowly transcribed genes (Li et al. 2007b; Cheung et al. 2008; Lickwar et al. 2009). Therefore, it is possible that highly transcribed SRG1 may not be dependent on H3K36 methylation and subsequent histone deacetylation for protection from intragenic transcription, because of the frequent passage of RNA Pol II. Alternatively, short, highly transcribed genes may never establish this histone mark, since histone H3K36 methylation predominates toward the $3^{\prime}$ ends of transcribed genes (Pokholok et al. 2005). In support of this possibility, genome-wide analyses of K36 methylation indicate little K36 trimethylation at SRG1 (Pokholok et al. 2005).

In contrast to the characteristic transcription-dependent depletion of nucleosomes seen at protein-coding genes (Yuan et al. 2005; Lee et al. 2007), we show transcriptiondependent assembly of nucleosomes across intergenic $S R G 1$. How does one account for this apparent contradiction between nucleosome occupancy and transcription? Several recent studies have indicated that DNA sequences can either favor or refract nucleosome formation, thereby influencing genome-wide nucleosome positioning (Yuan et al. 2005; Ioshikhes et al. 2006; Segal et al. 2006; Peckham et al. 2007; Field et al. 2008; Kaplan et al. 2009). As has been proposed for yeast genes containing nucleosome-depleted promoter regions (Segal and Widom 2009|, one possibility is that the underlying DNA sequence of the SER3 promoter may normally disfavor nucleosome formation to facilitate transcription factor binding. Therefore, by reassembling nucleosomes after each passage of RNA Pol II, SRG1 transcription effec- tively maintains nucleosomes over DNA that is normally refractory to nucleosomes. Several observations support this possibility. First, the SER3 UAS region that is nucleosome-depleted in the absence of SRG1 transcription contains poly(dA:dT) tracts, a sequence motif that resists bending and thus disfavors nucleosome formation (Segal and Widom 2009). Second, the SER3 UAS sequence is predicted to have a low nucleosome-forming potential by an algorithm developed using comparative genomics (Ioshikhes et al. 2006). Finally, the SER3 UAS sequence failed to form a stable nucleosome in a genome-wide in vitro nucleosome reconstitution assay (Kaplan et al. 2009).

In $S$. cerevisiae, cells respond to changes in serine availability by rapidly inducing or repressing transcription of SER3. This response involves a dynamic competition between nucleosomes and transcription factors that is controlled by the transcription of SRG1 from intergenic ncDNA. Our findings raise the intriguing possibility that widespread transcription of ncDNA may impact genomewide chromatin architecture. In doing so, transcription of ncDNA may influence not only gene expression, but also other cellular processes that are dependent on proteinDNA interactions.

\section{Materials and methods}

\section{S. cerevisiae strains and media}

All S. cerevisiae strains used in this study (Supplemental Table S1) are isogenic with a $G A L 2^{+}$derivative of S288C (Winston et al. 1995). Strains were constructed using standard genetic crosses or by transformation (Ausubel et al. 1991). The C termini of RPB1 and SPT16 were tagged with 13 copies of the c-Myc epitope by PCR-mediated transformation of diploid strains using pFA6a-13myc-KanMX and pFA6a-13myc-HIS3MX, respectively (Longtine et al. 1998). The spt16-22 and spt16-23 alleles (Formosa et al. 2001) were integrated into a diploid strain by twostep gene replacement using SnaBI-digested pTF142-23 and pTF142-22 plasmids (kindly provided by T. Formosa, University of Utah, Salt Lake City, UT). The ser3 $\triangle U A S$ mutation was constructed by replacing $37 \mathrm{bp}$ of SER3 promoter sequence (from -228 to -198 ; SER3 ATG $=+1$ ) with an AvrII restriction site by QuikChange mutagenesis (Agilent Technologies) to yield pRM08 plasmid. The ser3 $\triangle U A S$ allele was then integrated into a diploid strain by two-step gene replacement using AfeI-digested pRM08. Several strains contain a KanMX-marked deletion of the SER33 gene, which is a paralog of SER3. Based on previous studies (Martens and Winston 2002; Martens et al. 2004) and the results presented in this study, the deletion of SER33 does not affect SER3 regulation. Strains were grown in the following media as indicated in the figure legends: YPD ( $1 \%$ yeast extract, $2 \%$ peptone, $2 \%$ glucose), YPgal ( $1 \%$ yeast extract, $2 \%$ peptone, $2 \%$ galactose), YPraf ( $1 \%$ yeast extract, $2 \%$ peptone, $2 \%$ raffinose), and SC with $1 \mathrm{mM}$ serine (SC + serine) or without serine (SC - serine) (Rose et al. 1990).

\section{Nucleosome scanning assay}

Nucleosome scanning experiments were performed using a method adapted from those described previously (Whitehouse and Tsukiyama 2006; Brickner et al. 2007; Lee et al. 2007). Cells were grown to $2 \times 10^{7}$ to $3 \times 10^{7}$ cells per milliliter and were 
treated with formaldehyde ( $2 \%$ final concentration) for $30 \mathrm{~min}$ at $30^{\circ} \mathrm{C}$ and then glycine ( $125 \mathrm{mM}$ final concentration) for $10 \mathrm{~min}$ at room temperature. Formaldehyde-treated cells $\left(1.2 \times 10^{9}\right)$ were harvested by centrifugation, washed with Tris-buffered saline, and then incubated in ZDB buffer $(50 \mathrm{mM}$ Tris $\mathrm{Cl}$ at pH 7.5, $1 \mathrm{M}$ sorbitol, $10 \mathrm{mM} \beta$-mercaptoethanol) containing $1.5 \mathrm{mg}$ of zymolase $20 \mathrm{~T}$ for $30 \mathrm{~min}$ at $30^{\circ} \mathrm{C}$ on a rocker platform. Spheroplasts were pelleted by low-speed centrifugation, gently washed with NP buffer $(1 \mathrm{M}$ sorbitol, $50 \mathrm{mM} \mathrm{NaCl}, 10 \mathrm{mM}$ Tris $\mathrm{Cl}$ at $\mathrm{pH} 7.4,5 \mathrm{mM} \mathrm{MgCl} 2,1 \mathrm{mM} \mathrm{CaCl} 2,0.075 \%$ NP-40, $1 \mathrm{mM}$ $\beta$-mercaptoethanol, $500 \mu \mathrm{M}$ spermidine), and resuspended in 1.8 $\mathrm{mL}$ of NP buffer. Samples were divided into six $300-\mu \mathrm{L}$ aliquots that were then digested with $0,1,2.5,5,10$, and $20 \mathrm{U}$ of MNase (Nuclease S7 from Roche) for $45 \mathrm{~min}$ at $37^{\circ} \mathrm{C}$. Digestions were stopped with $75 \mu \mathrm{L}$ of Stop buffer (5\% SDS, $50 \mathrm{mM}$ EDTA) and were treated with $100 \mu \mathrm{g}$ of proteinase $\mathrm{K}$ for $12-16 \mathrm{~h}$ at $65^{\circ} \mathrm{C}$. DNA was extracted by phenol/chloroform using PLG-H tubes (5 Prime), and was incubated with $50 \mu \mathrm{g}$ of RNase A for $1 \mathrm{~h}$ at $37^{\circ} \mathrm{C}$. DNA was re-extracted with phenol/chloroform, precipitated with an equal volume of isopropanol, washed with $80 \%$ ethanol, and resuspended in $100 \mu \mathrm{L}$ of TE. MNase digestions were evaluated by two methods. First, one-fifth of digested DNA was separated by gel electrophoresis. Second, previously characterized GAL1 promoter sequences (Lohr 1984; Brickner et al. 2007; Floer et al. 2010)—one within a positioned nucleosome (GAL1 NB), and a second adjacent region (GAL1 NUB) that is rapidly digested by MNase-were amplified by qPCR from MNase-treated and untreated samples. The MNase concentration that resulted in mostly mononucleosome-sized DNA (see Supplemental Fig. S1) with a GAL1 NUB/NB ratio of $<15 \%$ was subjected to further qPCR using tiled SER3 primer pairs (SER3-1 to SER3-41) (Supplemental Table S2). For each SER3 primer set, the amount of protected template was calculated as a ratio between MNase-digested and undigested samples and then normalized to the amount of protected GAL1 NB template. All nucleosome scanning assays were done in triplicate using at least two independent strains as indicated in the figure legends.

\section{Northern analysis}

Northern analysis was performed as described previously (Ausubel et al. 1991) on $20 \mu \mathrm{g}$ of total RNA isolated from cells grown to $1 \times$ $10^{7}$ to $2 \times 10^{7}$ cells per milliliter. DNA probes were generated by random prime-labeling PCR fragments for SER3 (ChrV: 324059324307), SRG1 (ChrV: 322258-322559), and SCR1 (ChrV: 441741442266). SCR 1 serves as a loading control, since its RNA levels are unaffected by the mutations and growth conditions used in this study.

\section{ChIP analysis}

For histone $\mathrm{H} 3, \mathrm{TBP}$, and Rpb1-C13myc ChIPs, cells were grown in YPD at $30^{\circ} \mathrm{C}$ to $1 \times 10^{7}$ to $2 \times 10^{7}$ cells per milliliter. For Gal4 ChIPs, cells were grown in YPraf at $30^{\circ} \mathrm{C}$ to $0.8 \times 10^{7}$ cells per milliliter, and then an additional $4 \mathrm{~h}$ at $30^{\circ} \mathrm{C}$ after addition of $2 \%$ galactose. Chromatin preparation and treatment were preformed as described previously (Shirra et al. 2005). Gal4, histone H3, TBP, and Rpb1-13myc were immunoprecipitated by incubating sonicated chromatin overnight at $4^{\circ} \mathrm{C}$ with $1 \mu \mathrm{L}$ of anti-GAL4 DBD antibody (sc-577, Santa Cruz Biotechnology), $5 \mu \mathrm{L}$ of antihistone $\mathrm{H} 3$ antibody (ab1791, Abcam), $2 \mu \mathrm{L}$ of anti-TBP antibody (kind gift from G. Prelich, Albert Einstein College of Medicine), and $4 \mu \mathrm{L}$ of anti-c-myc A-14 antibody (sc-789, Santa Cruz Biotechnology), respectively. Dilutions of input and immunoprecipitated DNA were subjected to qPCR. All ChIP signals were normalized to a control: either GAL1 NB template (histone H3
ChIP), TELVI template located within a telomeric region on chromosome VI (Gal4 ChIP), or "No ORF" template located within a region of chromosome $\mathrm{V}$ that lacks ORFs (Rpb1C13myc and TBP ChIPs). Details regarding the primers used for qPCR in each ChIP experiment are listed in Supplemental Table S2.

qPCR

All qPCR data were obtained using an ABI 7300 or StepOnePlus Real-Time PCR system, SYBR green reagents (Fermentas), and the primer sets listed in Supplemental Table S2. All calculations were performed using Pfaffl methodology for relative quantitation of real-time PCR (Pfaffl 2001).

\section{Acknowledgments}

We thank Karen Arndt, Andrea Duina, Fred Winston, and Travis Mavrich for critical reading of this manuscript prior to submission. We are grateful to Karen Arndt, Tim Formosa, Grant Hartzog, Greg Prelich, and Fred Winston for providing us with antibodies, strains, and plasmids used in this work. This work was supported by NIH grant GM080470, and by an award from Pittsburgh Life Sciences Greenhouse to J.A.M.

\section{References}

Adkins MW, Tyler JK. 2006. Transcriptional activators are dispensable for transcription in the absence of Spt6-mediated chromatin reassembly of promoter regions. Mol Cell 21: 405-416.

Albert I, Mavrich TN, Tomsho LP, Qi J, Zanton SJ, Schuster SC, Pugh BF. 2007. Translational and rotational settings of H2A.Z nucleosomes across the Saccharomyces cerevisiae genome. Nature 446: 572-576.

Ashe HL, Monks J, Wijgerde M, Fraser P, Proudfoot NJ. 1997. Intergenic transcription and transinduction of the human $\beta$-globin locus. Genes Dev 11: 2494-2509.

Ausubel FM, Brent R, Kingston RE, Moore DD, Seidman JG, Smith JA, Struhl KE. 1991. Current protocols in molecular biology. John Wiley and Sons, New York.

Belotserkovskaya R, Oh S, Bondarenko VA, Orphanides G, Studitsky VM, Reinberg D. 2003. FACT facilitates transcription-dependent nucleosome alteration. Science 301: 10901093.

Berretta J, Morillon A. 2009. Pervasive transcription constitutes a new level of eukaryotic genome regulation. EMBO Rep 10: 973-982.

Bird AJ, Gordon M, Eide DJ, Winge DR. 2006. Repression of $\mathrm{ADH} 1$ and $\mathrm{ADH} 3$ during zinc deficiency by Zap1-induced intergenic RNA transcripts. EMBO J 25: 5726-5734.

Brickner DG, Cajigas I, Fondufe-Mittendorf Y, Ahmed S, Lee PC, Widom J, Brickner JH. 2007. H2A.Z-mediated localization of genes at the nuclear periphery confers epigenetic memory of previous transcriptional state. PLOS Biol 5: e81. doi: 10.1371/ journal.pbio.0050081.

Bumgarner SL, Dowell RD, Grisafi P, Gifford DK, Fink GR. 2009. Toggle involving cis-interfering noncoding RNAs controls variegated gene expression in yeast. Proc Natl Acad Sci 106: 18321-18326.

Camblong J, Iglesias N, Fickentscher C, Dieppois G, Stutz F. 2007. Antisense RNA stabilization induces transcriptional gene silencing via histone deacetylation in S. cerevisiae. Cell 131: 706-717.

Camblong J, Beyrouthy N, Guffanti E, Schlaepfer G, Steinmetz LM, Stutz F. 2009. Trans-acting antisense RNAs mediate 
transcriptional gene cosuppression in S. cerevisiae. Genes Dev 23: 1534-1545.

Carrozza MJ, Li B, Florens L, Suganuma T, Swanson SK, Lee KK, Shia WJ, Anderson S, Yates J, Washburn MP, et al. 2005. Histone $\mathrm{H} 3$ methylation by Set 2 directs deacetylation of coding regions by $\mathrm{Rpd} 3 \mathrm{~S}$ to suppress spurious intragenic transcription. Cell 123: 581-592.

Cheung V, Chua G, Batada NN, Landry CR, Michnick SW, Hughes TR, Winston F. 2008. Chromatin- and transcriptionrelated factors repress transcription from within coding regions throughout the Saccharomyces cerevisiae genome. PLOS Biol 6: e277. doi: 10.1371/journal.pbio.0060277.

Costa FF. 2010. Non-coding RNAs: Meet thy masters. Bioessays 32: 599-608.

Dollard C, Ricupero-Hovasse SL, Natsoulis G, Boeke JD, Winston F. 1994. SPT10 and SPT21 are required for transcription of particular histone genes in Saccharomyces cerevisiae. Mol Cell Biol 14: 5223-5228.

Eriksson PR, Mendiratta G, McLaughlin NB, Wolfsberg TG, Marino-Ramirez L, Pompa TA, Jainerin M, Landsman D, Shen CH, Clark DJ. 2005. Global regulation by the yeast Spt10 protein is mediated through chromatin structure and the histone upstream activating sequence elements. Mol Cell Biol 25: 9127-9137.

Field Y, Kaplan N, Fondufe-Mittendorf Y, Moore IK, Sharon E, Lubling Y, Widom J, Segal E. 2008. Distinct modes of regulation by chromatin encoded through nucleosome positioning signals. PLoS Comput Biol 4: e1000216. doi: 10.1371/ journal.pcbi.1000216.

Formosa T, Eriksson P, Wittmeyer J, Ginn J, Yu Y, Stillman DJ. 2001. Spt16-Pob3 and the HMG protein Nhp6 combine to form the nucleosome-binding factor SPN. EMBO I 20: 35063517.

Floer M, Wang X, Prabhu V, Berrozpe G, Narayan S, Spagna D, Alvarez D, Kendall J, Krasnitz A, Stepansky A, et al. 2010. A RSC/nucleosome complex determines chromatin architecture and facilitates activator binding. Cell 141: 407-418.

Gelbart ME, Kuroda MI. 2009. Drosophila dosage compensation: A complex voyage to the $\mathrm{X}$ chromosome. Development 136: 1399-1410.

Goodrich JA, Kugel JF. 2009. From bacteria to humans, chromatin to elongation, and activation to repression: The expanding roles of noncoding RNAs in regulating transcription. Crit Rev Biochem Mol Biol 44: 3-15.

Greger IH, Aranda A, Proudfoot N. 2000. Balancing transcriptional interference and initiation on the GAL7 promoter of Saccharomyces cerevisiae. Proc Natl Acad Sci 97: 84158420.

Gribnau J, Diderich K, Pruzina S, Calzolari R, Fraser P. 2000. Intergenic transcription and developmental remodeling of chromatin subdomains in the human $\beta$-globin locus. Mol Cell 5: 377-386.

Harrison BR, Yazgan O, Krebs JE. 2009. Life without RNAi: Noncoding RNAs and their functions in Saccharomyces cerevisiae. Biochem Cell Biol 87: 767-779.

Hess D, Liu B, Roan NR, Sternglanz R, Winston F. 2004. Spt10dependent transcriptional activation in Saccharomyces cerevisiae requires both the Spt10 acetyltransferase domain and Spt21. Mol Cell Biol 24: 135-143.

Hirota K, Miyoshi T, Kugou K, Hoffman CS, Shibata T, Ohta K. 2008. Stepwise chromatin remodelling by a cascade of transcription initiation of non-coding RNAs. Nature 456: 130-134.

Hongay CF, Grisafi PL, Galitski T, Fink GR. 2006. Antisense transcription controls cell fate in Saccharomyces cerevisiae. Cell 127: 735-745.
Houseley J, Rubbi L, Grunstein M, Tollervey D, Vogelauer M. 2008. A ncRNA modulates histone modification and mRNA induction in the yeast GAL gene cluster. Mol Cell 32: 685695.

Ioshikhes IP, Albert I, Zanton SJ, Pugh BF. 2006. Nucleosome positions predicted through comparative genomics. Nat Genet 38: 1210-1215.

Jacquier A. 2009. The complex eukaryotic transcriptome: Unexpected pervasive transcription and novel small RNAs. Nat Rev Genet 10: 833-844.

Jamai A, Puglisi A, Strubin M. 2009. Histone chaperone spt16 promotes redeposition of the original h3-h4 histones evicted by elongating RNA polymerase. Mol Cell 35: 377-383.

Joshi AA, Struhl K. 2005. Eaf3 chromodomain interaction with methylated H3-K36 links histone deacetylation to Pol II elongation. Mol Cell 20: 971-978.

Kaplan CD, Laprade L, Winston F. 2003. Transcription elongation factors repress transcription initiation from cryptic sites. Science 301: 1096-1099.

Kaplan N, Moore IK, Fondufe-Mittendorf Y, Gossett AJ, Tillo D, Field Y, LeProust EM, Hughes TR, Lieb JD, Widom J, et al. 2009. The DNA-encoded nucleosome organization of a eukaryotic genome. Nature 458: 362-366.

Kapranov P, Willingham AT, Gingeras TR. 2007. Genome-wide transcription and the implications for genomic organization. Nat Rev Genet 8: 413-423.

Keogh MC, Kurdistani SK, Morris SA, Ahn SH, Podolny V, Collins SR, Schuldiner M, Chin K, Punna T, Thompson NJ, et al. 2005. Cotranscriptional set 2 methylation of histone $\mathrm{H} 3$ lysine 36 recruits a repressive $\mathrm{Rpd} 3$ complex. Cell 123: 593605.

Kim T, Buratowski S. 2009. Dimethylation of H3K4 by Set1 recruits the Set3 histone deacetylase complex to $5^{\prime}$ transcribed regions. Cell 137: 259-272.

Lee JT. 2009. Lessons from X-chromosome inactivation: Long ncRNA as guides and tethers to the epigenome. Genes Dev 23: $1831-1842$.

Lee JS, Shilatifard A. 2007. A site to remember: H3K36 methylation a mark for histone deacetylation. Mutat Res 618: 130-134.

Lee CK, Shibata Y, Rao B, Strahl BD, Lieb JD. 2004. Evidence for nucleosome depletion at active regulatory regions genomewide. Nat Genet 36: 900-905.

Lee W, Tillo D, Bray N, Morse RH, Davis RW, Hughes TR, Nislow C. 2007. A high-resolution atlas of nucleosome occupancy in yeast. Nat Genet 39: 1235-1244.

Li B, Carey M, Workman JL. 2007a. The role of chromatin during transcription. Cell 128: 707-719.

Li B, Gogol M, Carey M, Pattenden SG, Seidel C, Workman JL. $2007 \mathrm{~b}$. Infrequently transcribed long genes depend on the Set2/Rpd3S pathway for accurate transcription. Genes Dev 21: 1422-1430.

Li L, Feng T, Lian Y, Zhang G, Garen A, Song X. 2009. Role of human noncoding RNAs in the control of tumorigenesis. Proc Natl Acad Sci 106: 12956-12961.

Lickwar CR, Rao B, Shabalin AA, Nobel AB, Strahl BD, Lieb JD. 2009. The Set2/Rpd3S pathway suppresses cryptic transcription without regard to gene length or transcription frequency. PLOS ONE 4: e4886. doi: 10.1371/journal.pone.0004886.

Lohr D. 1984. Organization of the GAL1-GAL10 intergenic control region chromatin. Nucleic Acids Res 12: 8457-8474.

Longtine MS, McKenzie A 3rd, Demarini DJ, Shah NG, Wach A, Brachat A, Philippsen P, Pringle JR. 1998. Additional modules for versatile and economical PCR-based gene deletion and modification in Saccharomyces cerevisiae. Yeast 14: 953-961. 
Martens JA, Winston F. 2002. Evidence that Swi/Snf directly represses transcription in S. cerevisiae. Genes Dev 16: 22312236.

Martens JA, Laprade L, Winston F. 2004. Intergenic transcription is required to repress the Saccharomyces cerevisiae SER3 gene. Nature 429: 571-574.

Martens JA, Wu PY, Winston F. 2005. Regulation of an intergenic transcript controls adjacent gene transcription in Saccharomyces cerevisiae. Genes Dev 19: 2695-2704.

Mason PB, Struhl K. 2003. The FACT complex travels with elongating RNA polymerase II and is important for the fidelity of transcriptional initiation in vivo. Mol Cell Biol 23: $8323-8333$.

Mazo A, Hodgson JW, Petruk S, Sedkov Y, Brock HW. 2007. Transcriptional interference: An unexpected layer of complexity in gene regulation. J Cell Sci 120: 2755-2761.

Mercer TR, Dinger ME, Mattick JS. 2009. Long non-coding RNAs: Insights into functions. Nat Rev Genet 10: 155-159.

Peckham HE, Thurman RE, Fu Y, Stamatoyannopoulos JA, Noble WS, Struhl K, Weng Z. 2007. Nucleosome positioning signals in genomic DNA. Genome Res 17: 1170-1177.

Pfaffl MW. 2001. A new mathematical model for relative quantification in real-time RT-PCR. Nucleic Acids Res 29: e45. doi: 10.1093/nar/29.9.e45.

Pheasant M, Mattick JS. 2007. Raising the estimate of functional human sequences. Genome Res 17: 1245-1253.

Pinskaya M, Gourvennec S, Morillon A. 2009. H3 lysine 4 diand tri-methylation deposited by cryptic transcription attenuates promoter activation. EMBO J 28: 1697-1707.

Pokholok DK, Harbison CT, Levine S, Cole M, Hannett NM Lee TI, Bell GW, Walker K, Rolfe PA, Herbolsheimer E, et al. 2005. Genome-wide map of nucleosome acetylation and methylation in yeast. Cell 122: 517-527.

Rao B, Shibata Y, Strahl BD, Lieb JD. 2005. Dimethylation of histone $\mathrm{H} 3$ at lysine 36 demarcates regulatory and nonregulatory chromatin genome-wide. Mol Cell Biol 25: 9447-9459.

Rinn JL, Kertesz M, Wang JK, Squazzo SL, Xu X, Brugmann SA, Goodnough LH, Helms JA, Farnham PJ, Segal E, et al. 2007. Functional demarcation of active and silent chromatin domains in human HOX loci by noncoding RNAs. Cell 129: $1311-1323$.

Rose M, Winston F, Hieter P. 1990. Methods in yeast genetics; A laboratory course manual. Cold Spring Harbor Laboratory Press, Cold Spring Harbor, NY.

Royo H, Cavaille J. 2008. Non-coding RNAs in imprinted gene clusters. Biol Cell 100: 149-166.

Schmitt S, Prestel M, Paro R. 2005. Intergenic transcription through a polycomb group response element counteracts silencing. Genes Dev 19: 697-708.

Schwabish MA, Struhl K. 2004. Evidence for eviction and rapid deposition of histones upon transcriptional elongation by RNA polymerase II. Mol Cell Biol 24: 10111-10117.

Segal E, Widom J. 2009. What controls nucleosome positions? Trends Genet 25: 335-343.

Segal E, Fondufe-Mittendorf Y, Chen L, Thastrom A, Field Y, Moore IK, Wang JP, Widom J. 2006. A genomic code for nucleosome positioning. Nature 442: 772-778.

Seila AC, Core LJ, Lis JT, Sharp PA. 2009. Divergent transcription: A new feature of active promoters. Cell Cycle 8: 25572564.

Sekinger EA, Moqtaderi Z, Struhl K. 2005. Intrinsic histoneDNA interactions and low nucleosome density are important for preferential accessibility of promoter regions in yeast. Mol Cell 18: 735-748.

Shirra MK, Rogers SE, Alexander DE, Arndt KM. 2005. The Snf1 protein kinase and Sit4 protein phosphatase have opposing functions in regulating TATA-binding protein association with the Saccharomyces cerevisiae INO1 promoter. Genetics 169: 1957-1972.

Struhl K. 2007. Transcriptional noise and the fidelity of initiation by RNA polymerase II. Nat Struct Mol Biol 14: 103-105.

Thompson DM, Parker R. 2007. Cytoplasmic decay of intergenic transcripts in Saccharomyces cerevisiae. Mol Cell Biol 27: 92-101.

van Bakel H, Nislow C, Blencowe BJ, Hughes TR. 2010. Most 'dark matter' transcripts are associated with known genes. PLoS Biol 8: e1000371. doi: 10.1371/journal.pbio.1000371.

Wang G, Cui Y, Zhang G, Garen A, Song X. 2009. Regulation of proto-oncogene transcription, cell proliferation, and tumorigenesis in mice by PSF protein and a VL30 noncoding RNA. Proc Natl Acad Sci 106: 16794-16798.

Whitehouse I, Tsukiyama T. 2006. Antagonistic forces that position nucleosomes in vivo. Nat Struct Mol Biol 13: 633640.

Winston F, Dollard C, Ricupero-Hovasse SL. 1995. Construction of a set of convenient Saccharomyces cerevisiae strains that are isogenic to S288C. Yeast 11: 53-55.

Wyrick JJ, Holstege FC, Jennings EG, Causton HC, Shore D, Grunstein M, Lander ES, Young RA. 1999. Chromosomal landscape of nucleosome-dependent gene expression and silencing in yeast. Nature 402: 418-421.

Xin H, Takahata S, Blanksma M, McCullough L, Stillman DJ, Formosa T. 2009. yFACT induces global accessibility of nucleosomal DNA without $\mathrm{H} 2 \mathrm{~A}-\mathrm{H} 2 \mathrm{~B}$ displacement. Mol Cell 35: 365-376.

Youdell ML, Kizer KO, Kisseleva-Romanova E, Fuchs SM, Duro E, Strahl BD, Mellor J. 2008. Roles for Ctk1 and Spt6 in regulating the different methylation states of histone $\mathrm{H} 3$ lysine 36. Mol Cell Biol 28: 4915-4926.

Yuan GC, Liu YJ, Dion MF, Slack MD, Wu LF, Altschuler SJ, Rando OJ. 2005. Genome-scale identification of nucleosome positions in S. cerevisiae. Science 309: 626-630. 


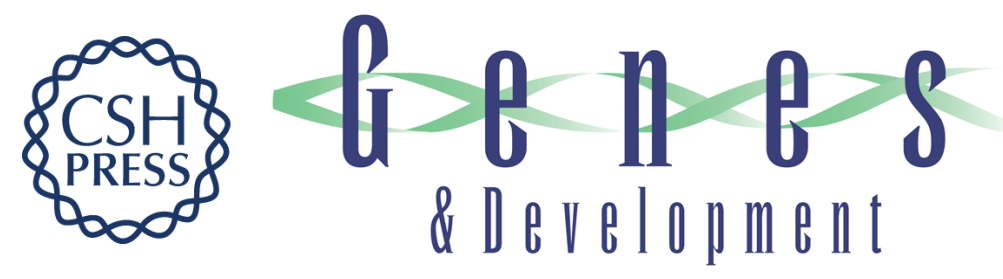

\section{Intergenic transcription causes repression by directing nucleosome assembly}

Sarah J. Hainer, Justin A. Pruneski, Rachel D. Mitchell, et al.

Genes Dev. 2011, 25: originally published online December 14, 2010

Access the most recent version at doi:10.1101/gad.1975011

\section{Supplemental http://genesdev.cshlp.org/content/suppl/2010/12/15/gad.1975011.DC1 Material}

References This article cites 77 articles, 27 of which can be accessed free at: http://genesdev.cshlp.org/content/25/1/29.full.html\#ref-list-1

\section{License}

Email Alerting

Service

Receive free email alerts when new articles cite this article - sign up in the box at the top right corner of the article or click here.

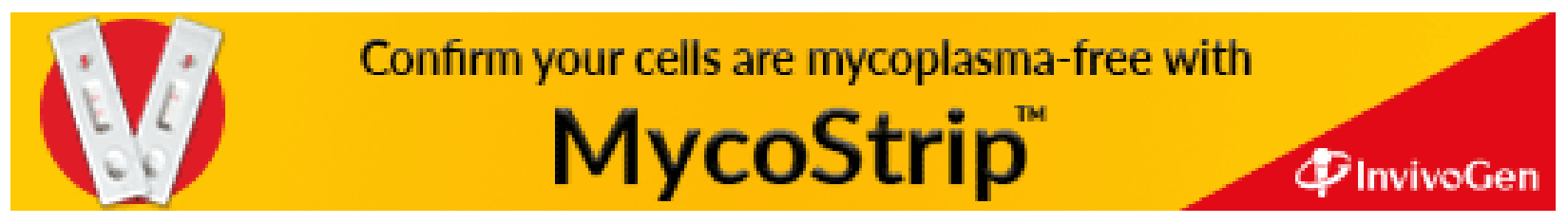

